\title{
Stressor controllability modulates fear extinction in humans
}

\section{Citation}

Hartley, Catherine A., Alyson Gorun, Marianne C. Reddan, Franchesca Ramirez, and Elizabeth A. Phelps. 2014. "Stressor Controllability Modulates Fear Extinction in Humans." Neurobiology of Learning and Memory 113 (September): 149-156. doi:10.1016/j.nlm.2013.12.003.

\section{Published Version}

doi:10.1016/j.nlm.2013.12.003

\section{Permanent link}

http://nrs.harvard.edu/urn-3:HUL.InstRepos:37308307

\section{Terms of Use}

This article was downloaded from Harvard University's DASH repository, and is made available under the terms and conditions applicable to Other Posted Material, as set forth at http:// nrs.harvard.edu/urn-3:HUL.InstRepos:dash.current.terms-of-use\#LAA

\section{Share Your Story}

The Harvard community has made this article openly available.

Please share how this access benefits you. Submit a story.

\section{Accessibility}




\title{
Stressor controllability modulates fear extinction in humans
}

\author{
Catherine A. Hartley ${ }^{1,2}$, Alyson Gorun ${ }^{3}$, Marianne C. Reddan ${ }^{4}$, Franchesca Ramirez ${ }^{1}$, and \\ Elizabeth A. Phelps ${ }^{1,5,6}$ \\ ${ }^{1}$ Department of Psychology, New York University, New York, NY 10003 \\ ${ }^{2}$ Sackler Institute for Developmental Psychobiology, Weill Cornell Medical College, New York, NY \\ 10003 \\ ${ }^{3}$ New York University School of Medicine, New York, NY 10016 \\ 4Department of Psychiatry, Mount Sinai School of Medicine, New York, NY 10029 \\ ${ }^{5}$ Center for Neural Science, New York University, New York, NY 10003 \\ ${ }^{6}$ Nathan Kline Institute, Orangeburg, NY 10962
}

\begin{abstract}
Traumatic events are proposed to play a role in the development of anxiety disorders, however not all individuals exposed to extreme stress experience a pathological increase in fear. Recent studies in animal models suggest that the degree to which one is able to control an aversive experience is a critical factor determining its behavioral consequences. In this study, we examined whether stressor controllability modulates subsequent conditioned fear expression in humans. Participants were randomly assigned to an escapable stressor condition, a yoked inescapable stressor condition, or a control condition involving no stress exposure. One week later, all participants underwent fear conditioning, fear extinction, and a test of extinction retrieval the following day. Participants exposed to inescapable stress showed impaired fear extinction learning and increased fear expression the following day. In contrast, escapable stress improved fear extinction and prevented the spontaneous recovery of fear. Consistent with the bidirectional controllability effects previously reported in animal models, these results suggest that one's degree of control over aversive experiences may be an important factor influencing the development of psychological resilience or vulnerability in humans.
\end{abstract}

\section{Keywords}

Fear conditioning; Extinction; Controllability; Resilience

\footnotetext{
(c) 2013 Elsevier Inc. All rights reserved.

Corresponding Author: Elizabeth Phelps, 6 Washington Place, New York, NY 10003, Phone: (212) 998-8337, liz.phelps@nyu.edu. Publisher's Disclaimer: This is a PDF file of an unedited manuscript that has been accepted for publication. As a service to our customers we are providing this early version of the manuscript. The manuscript will undergo copyediting, typesetting, and review of the resulting proof before it is published in its final citable form. Please note that during the production process errors may be discovered which could affect the content, and all legal disclaimers that apply to the journal pertain.
} 


\section{Introduction}

Instrumental control over an aversive experience, or the ability to influence its intensity, duration, onset, or termination, has long been recognized as a critical factor determining its behavioral and physiological impact on an organism (Brady, 1958; Mineka and Hendersen, 1985; Rotter, 1966; Seligman and Maier, 1967). The importance of stressor controllability was initially evidenced by the demonstration that exposure of an animal to inescapable shocks yielded impairment in subsequent avoidance learning that was not observed in animals exposed to identical but escapable shocks (Seligman and Maier, 1967). Subsequent studies revealed that uncontrollable stress exposure results in a host of other behavioral and physiological consequences including neophobia, reduced social interaction, decreased social dominance and aggression, heightened immobility in a forced swim task, decreased food and water consumption, formation of ulcers, and the potentiation of fear conditioning (see Maier and Watkins, 2005 for a review). As many of these behaviors mirror the symptoms of depression and anxiety, it has been proposed that exposure to uncontrollable stress may play an important role in the etiology of these disorders (Maier and Watkins, 2005; Weiss and Simson, 1986). While studies of uncontrollable stress reveal a broad array of deleterious effects, an equally striking result is that controllable stress yields none of these consequences, but in contrast, appears to promote behavioral resilience.

Stressor controllability effects are typically studied using a triadic design. One group of subjects is exposed to aversive reinforcement (such as electric shock) that they are able avoid or escape via the performance of an instrumental avoidance response. A second group is yoked to the first group, receiving reinforcement that is identical in intensity and duration, but that they cannot control through any action of their own. A third control group receives no exposure to the stressor. All three groups then take part in an identical generalization task through which the effects of prior experience are assessed. Importantly, this design enables the distinction between effects of stress exposure and the degree to which controllability modulates these effects. A host of recent studies employing this design have found that subjects exposed to escapable stress exhibit performance in the generalization task comparable or even superior to that of unstressed controls (Maier and Watkins, 2010). These findings suggest that the ability to exercise control over a stressor blunts its detrimental effects. Furthermore, several studies report that animals exposed to escapable stress fail to show the typical neurochemical and behavioral consequences of later inescapable stress (Amat, Aleksejev, Paul, Watkins, and Maier, 2010; Amat, Paul, Zarza, Watkins, and Maier, 2006; Williams and Maier, 1977), suggesting that escapable stress yields long-lasting neurobiological changes that immunize an organism to subsequent aversive experiences. Thus, contrary to the conventional notion that stress is physiologically harmful, these studies indicate that stressful experience may actually play a critical role in fostering resilience, provided that it is controllable.

Recent studies in rodents suggest that stressor controllability powerfully influences the expression of conditioned fear (Baratta, Christianson, Gomez, Zarza, Amat, Masini, Watkins, and Maier, 2007; Cain and LeDoux, 2007; Rau, DeCola, and Fanselow, 2005). In one such study (Baratta et al., 2007), inescapable stress potentiated and escapable stress mitigated subsequent conditioned fear expression during fear acquisition, fear extinction 
learning, and a test of extinction retrieval, with respect to unstressed control animals. These findings suggest a mechanism by which individual variation in life experiences may modulate the expression of learned threat and safety associations; however, such effects have not presently been demonstrated in humans. In this study, we explore whether stressor controllability in humans yields the bidirectional effects on conditioned fear expression previously observed in animal models. As dysregulated fear expression is proposed to play a mechanistic role in the etiology of anxiety disorders (Lissek, Powers, McClure, Phelps, Woldehawariat, Grillon, and Pine, 2005; Milad and Quirk, 2012; Mineka and Zinbarg, 2006), such a finding would implicate stressor controllability as an important experiential mechanism underlying psychological resilience or vulnerability.

\section{Materials and Methods}

\subsection{Participants}

Participants in both experiments were recruited at New York University. All participants gave their informed consent to take part in the study and were paid for their participation.

Experiment 1: One hundred and two participants (75 female), aged 18-50 (mean age $=22.8)$ were randomly assigned to one of three experimental conditions, an escapable stress (ES) condition, a yoked inescapable stress (IS) condition, or a control condition. Twenty-five participants in the ES condition who did not learn to perform the necessary avoidance response to criterion were dismissed after the initial session, as the expected effects of control over a stressor would be dependent upon this learning. In addition, 16 participants' data (4 control, $5 \mathrm{ES}$, and 7 IS participants) were excluded from analysis due to failure to show a measurable increase skin conductance response to the conditioned stimuli during the fear conditioning task (non-responders), preventing the use of this measure as in index of learning. Two yoked IS condition participants were excluded due to their yoked ES counterpart being a non-responder. The remaining 59 participants ( 41 female), aged 18-43 (mean age $=21.9$ ), are included in the analyses presented here. Of these participants, 20 were in the ES condition, 20 were in the IS condition, and 19 were in the control condition.

Experiment 2: As a large proportion of participants randomly assigned to the ES condition in experiment 1 failed to learn the avoidance response in the stressor task, it is possible any differences in fear conditioning observed in this group could stem from selection bias and not stressor controllability. To address this potential confound, we conducted a follow-up control experiment in which the order to the two tasks was reversed in order to determine whether fear conditioning differed between participants subsequently classified as learners and non-learners in the avoidance task. Forty-one participants (24 female), aged 18-53 (mean age $=23.3$ ) took part in a follow-up control study. Twenty-three of these participants learned the avoidance response in the stressor task (learners) and 18 did not (non-learners) Six participants' data were excluded from analysis due to being non-responders. One additional participant was excluded due to a failure to exhibit any skin conductance response to over one-third of the shock presentations, preventing the use of these measurements for the normalization of the CS-evoked SCRs. The remaining 34 participants ( 20 female), aged 18-53 (mean age $=23.4$ ) were included in the present analysis, and included 20 learners and 14 non-learners. Due to a technical error, physiological recording terminated early on day 1 
for three of these participants (1 non-learner, 2 learners), preventing the measurement of their SCRs at the late extinction timepoint. However acquisition and extinction retrieval measures for these participants are included in the present analysis.

\subsection{Experimental Paradigms}

In experiment 1, participants were assigned to an escapable stress (ES) condition, a yoked inescapable stress (IS) condition, or a control condition. Participants in the ES and IS conditions took part in three experimental sessions: a stressor task, followed seven days later by a two-day fear conditioning paradigm. Participants in the control condition only took part in the fear conditioning sessions and had no exposure to an initial stressor task. E-Prime software (Psychology Software Tools) was used to perform stimulus presentation and response collection in both tasks.

In experiment 2, a separate cohort of participants took part in a follow-up study in which the order of the two tasks in experiment 1 was reversed. Participants completed the two-day fear conditioning task followed two to three days later by the controllable variant of the stressor task. This enabled the assessment of whether fear conditioning and extinction differed between participants subsequently classified as learners and non-learners in the avoidance task, which would suggest that selection of learners for the ES group in experiment 1 might bias the results. In addition, by comparing fear conditioning in learners across both experiments, we can test whether the order in which the tasks were completed appears to influence fear conditioning, a key assumption motivating experiment 1.

Shock administration, skin conductance recording, and the task paradigms were identical across the two studies.

2.2.1 Shock Administration-At the start of the initial experimental session, participants determined the level of a mild electric shock (200ms, 50 pulses/s) via a work-up procedure in which the shock level was gradually increased to a level that the participant deemed to be "uncomfortable, but not painful." This same shock level was used in all subsequent sessions. The maximum shock level administered was $60 \mathrm{mV}$. Shocks were delivered via a stimulator (Grass Instruments) connected to a bar electrode attached to the wrist of the participant's dominant hand. The stimulator was charged by a stabilized current using magnetically shielded and RF-grounded cable leads.

2.2.2 Skin Conductance Recording - At the start of each session, shielded $\mathrm{Ag}-\mathrm{AgCl}$ recording electrodes were filled with a $\mathrm{NaCl}$ electrolyte gel and attached to the second and third fingers of the participant's non-dominant hand. Skin conductance data were continuously recorded throughout all three sessions at a rate of 200 samples per second using a Biopac Systems skin conductance module and AcqKnowledge software (Biopac Systems).

2.2.3 Stressor Task-Participants assigned to the ES or yoked IS condition took part in a task in which they were exposed, respectively, to a controllable or uncontrollable variant of the stressor task. All participants in the stressor task were instructed that the task consisted of a series of trials in which a grid would be shown on the screen. They were told that 
shocks would only be administered while the grids were on the screen and that they would be able to use the arrow keys on the computer keyboard to "explore different actions" in the grid trials. No explicit instructions were provided as to the goal of the task.

A graphical depiction of a trial in the stressor task is shown in Figure 1a. Each trial began with a 4 second anticipatory period, during which they saw a white screen framed in yellow. The frame color then changed to purple and a $5 \times 5$ cell grid appeared in the center of the screen with a red circle positioned in one of the cells along the edge of the grid. The position of the circle moved within the grid in correspondence with each directional arrow key press made by the participant. Each trial lasted 6 seconds. Participants in the ES condition received shocks every 2 seconds during this period ( 3 shocks per trial) until they moved the ball to one of the 5 cells comprising the edge of the grid opposite its starting position. Performance of this "shuttle" escape response terminated all further shocks in the trial. Corner cells were not used as starting positions to avoid ambiguity as to the correct direction of the escape response. Following a shuttle response, the position of the red circle was frozen in the final grid cell and the screen frame was colored blue for the remainder of the 6 second trial. These features served as additional indications that the participant had achieved the goal state. Participants completed 15 trials with randomized starting positions. Each trial was followed by a 6-8 second intertrial interval.

Participants who escaped or avoided shocks on at least one-third of trials were considered to have learned. In experiment 1 , we used the responses of those participants who learned successfully to create yoked stimulus presentations for participants in the uncontrollable IS condition. IS condition participants were able to use the arrows to move the red circle, however their actions had no effect on their reinforcement. Delivery of shock on each trial replicated precisely that of their yoked ES counterpart. Each IS participant received the identical number, sequence, and timing of shocks as their yoked counterpart within the ES group, ensuring that any observed differences between the ES and IS groups in subsequent fear conditioning could not stem from differences in number of shocks received. For participants in the ES condition who did not learn to perform the escape response, the session terminated following the delivery of the $30^{\text {th }}$ shock and they were excluded from the remaining sessions of the study.

An optimal escape response in the task required a participant to make only four key presses to traverse the grid. For participants in the ES condition, we calculated the mean path length (number of valid key presses) of all successful escape responses as an objective measure of instrumental avoidance learning.

Skin conductance data from the stressor task was low-pass filtered and smoothed. We measured the base to peak magnitude of any skin conductance response (SCR) beginning between $.5 \mathrm{~ms}$ after the onset of the trial, and the end of the $4 \mathrm{~s}$ anticipatory period. These SCR values were then square-root transformed to normalize the distribution of responses and divided by the individual's largest anticipatory SCR in order to enable between-subject comparisons. We did not analyze SCRs during the grid phase of each trial due to the design of our stressor task. Stimulus-evoked skin conductance responses in humans occur on a relatively slow timescale, typically taking between 500 and 4000ms to begin, and 1-2 
additional seconds for the full phasic trough-to-peak response to be completed (Dawson et al., 2007). The short interval between successive shocks during the trial made it difficult to distinguish anticipatory responses from responses to the shock, as well as the full magnitude of any such responses.

2.2.4 Fear Conditioning-In experiment 1, participants in the stressor task returned one week later and took part in a two-day partial reinforcement fear conditioning paradigm (Figure 1b), which took place in a different testing room than the stressor task. A third group of control participants also took part in the fear conditioning, with no prior exposure to the stressor task. In experiment 2, fear conditioning took place before the stressor task.

During the fear acquisition phase, participants were shown two colored abstract shapes, one of which (the CS+) was paired with the shock (the unconditioned stimulus or US) on 50\% of presentations. The other shape (the CS-) was never paired with the US. Each CS was presented for $4 \mathrm{~s}$, and shocks (200ms in duration) were presented during the final $200 \mathrm{~ms}$ of each reinforced CS presentation, thus co-terminating with the CS. The skin conductance response to each stimulus was recorded throughout the session. An extinction phase, in which the CS+ was no longer reinforced, followed immediately after acquisition.

Participants returned the following day for another extinction session, which enabled us to assess the retention of extinction learning from the previous day.

Trials in all phases were grouped into "blocks", such that acquisition consisted of 3 blocks of 4 unreinforced CS+, 4 CS-, and 4 reinforced CS+ trials, the initial extinction phase consisted of 6 blocks of 4 unreinforced CS+ and 4 CS- trials, and the extinction retrieval phase consisted of 4 blocks of 4 unreinforced CS+ and 4 CS- trials. Trials were presented in one of two pseudo-randomized trial orders, which were counterbalanced across subjects.

The colors of the stimuli designated as the CS+ and CS- were reversed in each of these trial orderings.

Skin conductance data from the fear conditioning task was low-pass filtered and smoothed. The base to peak change in SCR in the .5 to $4.5 \mathrm{sec}$ window following the onset of each CS was recorded for each trial. SCR values were then square-root transformed to normalize the distribution of responses and divided by their mean unconditioned SCR to the shock to enable between-subject comparisons. The difference between the mean CS+ SCR values and CS- SCR values in each block served as our discriminative conditioned fear response measure. Only CS+ presentations that were not reinforced with shock were included in the conditioned response measure. Given the slow timescale of SCR responses, shocks on reinforced CS+ trials often occur before CS-evoked responses reach their peak. Thus, we exclude reinforced trials from analysis and use only those trials in which the full CS-evoked SCR can be assessed.

2.2.5 Self-Report Measures-After completing the final fear conditioning session on day two, participants completed the Internal Control Index (Duttweiler, 1984), a psychological measure indexing "locus of control", or the degree to which one believes they can control salient everyday life events (Lefcourt, 1982; Rotter, 1966). Participants in the ES and IS groups also completed a post-experimental survey gauging their perception of control 
in the stressor task (one ES participant's responses on this survey were lost due to a technical error).

\section{Results}

\subsection{Effects of Variable Shock Level and Number on Fear Expression}

A yoked design ensured that each participant in an escapable stress (ES) and inescapable stress (IS) yoked pair received the same number of shocks during the stressor task.

However, each individual was able to set the shock at a level that they deemed to be subjectively uncomfortable, and differences in these levels could potentially modulate fear expression. A one-way ANOVA revealed no significant group differences in shock levels $(\mathrm{F}(2,56)=.223 ; \mathrm{p}=.801)$ between control $(\mathrm{M}=41.1$ volts, $\mathrm{SD}=9.03), \mathrm{ES}(\mathrm{M}=39.0$ volts, $\mathrm{SD}=8.94)$ and $\mathrm{IS}(\mathrm{M}=39.3$ volts, $\mathrm{SD}=12.78)$ participants, suggesting that this was not a confounding factor in our subsequent analyses. Furthermore, across all participants, there was no significant correlation between shock level and participants' mean conditioned response across all phases fear conditioning $(r(57)=-.123, p=.354)$. In addition, although number of shocks received in the stressor task varied across participants within the ES and IS groups, this variable shock number did not correlate with conditioned response measures during any phase of fear conditioning within either group (all $p$ values $>.5$ ).

\subsection{Skin Conductance Responses During the Stressor Task}

Comparison of skin conductance responses in the escapable and inescapable stress group during the 4 second anticipatory period that preceded the grid presentation for each avoidance trial revealed no differences in the magnitude of anticipatory responses $(t(38)=$. $225, p=.823)$.

\subsection{Controllability Effects on Fear Expression}

We first confirmed that all groups showed evidence of fear learning. One-sample t-tests confirmed that conditioned responses (mean SCR to the CS+ minus CS-) in all groups during late acquisition (mean of the second and third blocks of acquisition) were significantly different than 0 (all groups $\mathrm{p}<.005$ ) and fear expression did not differ between groups $(\mathrm{F}(2,56)=.033, \mathrm{p}=.968)$

We then examined whether stressor controllability modulated fear expression at three key phases of fear conditioning, late acquisition, late extinction, and day two extinction retrieval. Based on previous findings in rodents using a similar design (Baratta et al., 2007), we anticipated a bidirectional effect of stressor controllability on fear expression, in which controllable stress in the ES group would mitigate fear, and uncontrollable stress in the IS group would potentiate fear, compared to unstressed controls. We tested for this effect by coding a linear group variable with ES participants assigned a value of 1, controls assigned the value 2 , and IS participants assigned the value 3 . We conducted simple linear regression to test whether this variable accounted for differences in the mean conditioned response during acquisition (mean of the second and third blocks), the final block of extinction, and the first block of extinction retrieval (Figure 2). There was a significant linear effect of group on extinction recall $(\beta=.303, p=.020)$, but not acquisition $(\beta=-.005, p=.968)$ or late 
extinction $(\beta=.092, p=.487)$. Pairwise t-tests between groups confirmed that fear expression during extinction retrieval was significantly higher in IS versus ES participants $(t(38)=2.40, p=.021)$, but revealed no other significant group differences. One-sample ttests showed that while conditioned responses in both the control and IS groups were significantly greater than zero during the extinction retrieval test (control: $t(18)=2.54, p=$. 020; IS: $t(19)=4.511, p=.00024)$, ES participants showed no significant fear expression $(t(19)=.962, p=.348)$.

We then conducted paired t-tests to examine extinction learning and retention within each group. Conditioned responses in the ES group were significantly lower during late extinction than in late acquisition $(\mathrm{t}(19)=2.103, \mathrm{p}=.049)$ and remained significantly diminished at the start of the following day $(\mathrm{t}(19)=2.821, \mathrm{p}=.011)$, demonstrating successful extinction learning and retention. Control participants showed a marginally significant reduction in fear from late acquisition to late extinction $(\mathrm{t}(18)=1.738, \mathrm{p}=.099)$, however fear expression between late acquisition and the day two retrieval test did not differ $(\mathrm{t}(18)=.946, \mathrm{p}=.357)$. Fear expression in IS group participants did not differ significantly between late acquisition and late extinction $(\mathrm{t}(19)=1.237, \mathrm{p}=.231$, or late acquisition and retrieval test $(\mathrm{t}(19)=-$. $257, \mathrm{p}=.800$ ), revealing a deficit in extinction learning on day one and continued high fear expression the following day. Although no groups showed a significant change in fear expression from day one late extinction to they the day two retrieval test, we observed a general pattern of linearly increasing fear expression, with ES participants showing a small reduction in fear expression $(\mathrm{t}(19)=-.045, \mathrm{p}=.965)$, control participants showing a small increase $(\mathrm{t}(18)=.817, \mathrm{p}=.424)$, and IS participants showing a larger increase $(\mathrm{t}(19)=$ $1.184, \mathrm{p}=.251)$.

\subsection{Subjective and Objective Measures of Controllability}

Participants in the ES and IS groups rated their degree of confidence that they were able to control the occurrence of shocks on a 5-point scale where 1 indicated that they were "not at all confident" and 5 indicated that they were "very confident". Providing evidence of manipulation of participants' perceptions of control, the self-reported degree of control in the ES group $(\mathrm{M}=3.63, \mathrm{SD}=1.606)$ was significantly higher than in the IS group $(\mathrm{M}=2.45$, $\mathrm{SD}=1.432)(t(37)=2.43, p=.020)$. However, participants' self-report data indicates that ES participants were not, on the whole, certain that they were in control.

As an objective measure of instrumental learning in the stressor task, we calculated the length of the path travelled in the grid on each successful trial in the stressor task for all ES participants, with 4 being the minimum number of steps needed to terminate or prevent shocks on each trial. Mean path lengths ranged from 4.8 to $9.77(\mathrm{M}=7.05, \mathrm{SD}=1.72)$, demonstrating substantial variation in ES participants' instrumental learning in the stressor task.

Because our manipulation was not equally effective at inducing a clear sense of control for all ES participants, we hypothesized that the effects of ES on conditioned fear responses might depend upon participants' reported perceptions of control or their objective learning in the stressor task. Each participant was asked to describe the specific response, if any, that they performed to terminate the shock and to state their degree of certainty that this response 
was correct (where $1=$ very unsure and $5=$ very sure). A simple linear regression revealed that this subjective response confidence measure predicted reduced fear expression during extinction retrieval across ES participants $(\beta=-.486, p=.035)$, as well as a lower overall mean conditioned response across all conditioning blocks ( $\beta=-.442, p=.058)$ (Figure 3). This suggests that controllable stress was more effective at diminishing fear expression across all phases of conditioning in those individuals who were most certain that knew how to control the shock. Mean path lengths in the stressor task correlated significantly with this subjective measure of learning $(r=-.474, p=.040)$, however there was no significant relationship between mean path length and conditioned responses during extinction retrieval ( $\beta=.268, p=.253$ ). This suggests that subsequent fear expression was more directly influenced by ES participants' subjective appraisals on control than their objective learning performance.

The psychological construct of "locus of control" is a relatively stable individual trait indexing whether individuals conceive of positive and negative environmental events as predominantly under their own "internal" control or determined by external factors (Lefcourt, 1982; Rotter, 1966). Prior experience with controllable and uncontrollable life events is proposed to shape individual locus of control beliefs. We administered the Internal Control Index (ICI; (Duttweiler, 1984)) to assess individual locus of control with the hypothesis that this measure might account for individual variation in both stressor task learning or fear expression during conditioning. A one-way ANOVA revealed no difference in ICI scores between ES, IS and control groups $(\mathrm{F}(2,56)=.034, \mathrm{p}=.967)$, as expected given the random group assignment of participants. Among ES participants, higher ICI scores predicted shorter mean path length, $(r=-.493, \mathrm{p}=.027)$ (Figure 4$)$, indicating that individuals with a more internal locus of control exhibited better instrumental avoidance learning. There was no significant relationship between ICI scores and fear expression across all participants or in the ES group alone.

\subsection{Fear Conditioning in Avoidance Learners and Non-Learners}

Conditioned responses during late acquisition, late extinction and extinction retrieval in the full cohort of participants in experiment 2 did not differ significantly from the control sample in experiment 1 , who also performed the conditioning task with no prior shock exposure. Comparison of the CRs of individuals who learned the avoidance task versus nonlearners also revealed no significant differences at any timepoint (all $p$ values > .28), suggesting that there are not apparent differences in fear conditioning as a function of instrumental learning ability.

\subsection{Controllability and Fear Conditioning Task Order Effects}

Comparison of conditioned responses in the cohorts of stressor task learners from experiment 1 and experiment 2 revealed no differences during late acquisition $(t(38)=.784$, $p=.438)$ or late extinction $(t(36)=1.166, p=.251)$, however fear expression was significantly lower during extinction retrieval $(t(38)=2.172, p=.036)$ in participants for whom fear conditioning occurred after the controllable stressor, consistent with our interpretation that stressor controllability diminished subsequent fear expression for these individuals. 


\section{Discussion}

Our data suggest that degree of instrumental control over a stressor modulates subsequent fear expression in humans. Consistent with reported findings in animal models (Baratta et al., 2007), we observed evidence of bidirectional effects of stressor controllability, with inescapable stress (IS) potentiating and escapable stress (ES) diminishing conditioned fear expression one week later. Whereas ES participants exhibited successful extinction and an absence of fear recovery, fear expression in IS participants was not significantly reduced through extinction learning and remained elevated the following day. Reflecting an additional cognitive factor that may be unique to humans, modulation of fear expression in ES participants depended on their subjective beliefs about their degree of control. Our data support recent evidence from animal studies (Baratta et al., 2007; Cain and LeDoux, 2007), suggesting that the exercise of instrumental control in an aversive context might facilitate extinction learning and retention through activation of common neural circuitry (Amat, Baratta, Paul, Bland, Watkins, and Maier, 2005; Baratta, Lucero, Amat, Watkins, and Maier, 2008; Quirk and Mueller, 2008), preventing the return of fear.

The previous study in rodents upon which this experiment was modeled observed clear bidirectional effects of stressor controllability on fear expression during acquisition, extinction, and a retrieval test (Baratta et al., 2007), whereas effects of control in our study were only apparent during extinction learning and retrieval. IS and ES participants' reports of perceived control, or lack thereof, were variable, and the effectiveness of our controllability manipulation may have been compromised by these differences in participants' subjective appraisals of instrumental control. Our stressor task required that subjects learn to "shuttle" across a grid to terminate shocks. Avoidance path data from the task suggest that even some participants who met our learning criterion employed suboptimal avoidance responses (e.g. circling around the edges of the grid), potentially undermining their subjective sense of control. Furthermore, unlike the analogous animal paradigms in which yoked inescapable animals have no ability to perform any putatively instrumental response, our IS subjects were able to move the ball within the grid. This opportunity to perform actions even with no reinforcement contingency may have encouraged the formation of illusory impressions of control. Subjective appraisals of instrumental control among ES participants predicted reduced fear expression across all phases of fear conditioning. This suggests that a modified controllability manipulation yielding more definitive perceptions of control might produce a stronger and more generalized modulation of fear expression. Conversely, this correlation could reflect a preexisting relationship between a more trait-like subjective belief in one's learning ability or the efficacy of one's actions and fear reactivity. Future studies indexing such traits prior to fear conditioning might clarify the predictive nature of such individual differences.

Demonstrating a similar relationship between subjective reports and objective task performance, ES participants' scores on the Internal Control Index, reflecting their belief that salient life events are typically controllable, correlated with their success at avoidance learning. Such internal locus of control beliefs are thought to be established through previous experiences of control over aversive and appetitive reinforcement (Lefcourt, 1982; Rotter, 1966). If this self-report measure indeed reflects historical controllability of life 
events, our data would support previous findings in animal models demonstrating that lack of controllability impairs subsequent avoidance learning (Maier and Watkins, 2005). Persistent behavioral effects of stressor controllability may bias organisms toward coping mechanisms that are best suited to their environment (Maier and Watkins, 2010). Experiences of control might facilitate active responses to subsequent stressors, downregulating the typical stress response so that goal-directed instrumental actions can be employed. In contrast, exposure to uncontrollable stress may facilitate passive forms of coping that involve behavioral inhibition, conservation of energy, and preparation for aversive stimulation (e.g. freezing, endogenous opioid release). A generalized bias toward behavioral inhibition in response to threat may be adaptive in an environment that affords little opportunity for control, however it may limit the exploration and discovery of rewarding instrumental actions in controllable environments (Huys and Dayan, 2009).

Research in animal models has begun to elucidate the neurocircuitry underlying stressor controllability effects. IS elicits increased serotonin release from the dorsal raphe nucleus (DRN), which is thought to underlie its behavioral effects (Amat, Matus-Amat, Watkins, and Maier, 1998). DRN neurons are sensitized by IS (Amat et al., 1998), potentiating reactivity to even mild subsequent stressors (Maier, Grahn, Kalman, Sutton, Wiertelak, and Watkins, 1993; Maier, Grahn, and Watkins, 1995). In contrast, ES does not yield heightened DRN activity (Amat et al., 2005). The ventromedial prefrontal cortex (vmPFC) receives the convergent somatomotor and sensory input that could enable detection of instrumental control and sends strong projections to inhibitory interneurons in the DRN that appear to downregulate its activity during ES (Amat et al., 2005; Amat, Paul, Watkins, and Maier, 2008; Peyron, Petit, Rampon, Jouvet, and Luppi, 1998). ES yields plasticity that enables subsequent stressors to reactivate the vmPFC, yielding resilience to stress even in the absence of control (Amat et al., 2008; Amat et al., 2006). The bidirectional modulation of conditioned fear expression by IS and ES may be effected via connections from the DRN and vmPFC to the amygdala. The DRN projects directly to the amygdala and may potentiate fear expression following IS (Maier et al., 1995). The infralimbic (IL) and prelimbic (PL) subregions of the vmPFC are both activated by ES (Baratta, Zarza, Gomez, Campeau, Watkins, and Maier, 2009) and may contribute independently to reducing fear expression. Projections from the PL to inhibitory cells within the DRN appear to prevent its activation by stress (Baratta et al., 2009). Projections from the IL to the intercalated cell masses within the amygdala might directly inhibit central nucleus activity, which mediates fear expression (Milad and Quirk, 2012). The IL region is critically involved in active avoidance learning (Moscarello and LeDoux, 2013), as well as in the acquisition, consolidation and retrieval of fear extinction memory (Milad and Quirk, 2012; Quirk and Mueller, 2008; Sierra-Mercado, Padilla-Coreano, and Quirk, 2011). The robust effect of controllable stress on fear extinction observed here suggests that activation of the IL induced by avoidance learning in ES participants may facilitate the acquisition and retention of extinction learning.

In this study, we demonstrated that instrumental control over receipt of shock reduced subsequent expression of a conditioned fear of shock. Although the bidirectional effects of prior shock exposure observed here in the ES and IS participants (versus a unidirectional habituation or sensitization) suggest that controllability played a critical modulatory role, our use of the same type of aversive stimulus in both experimental tasks confounds any 
assumption about the broader implications of these findings. Everyday life stressors, instead of posing physical harm, are often social or cognitive in nature. The exercise of control over such stressors might involve actions that alter threatening social contexts (e.g. inviting a friend to accompany you to an event where you won't know anyone) or alleviate unpleasant cognitive states (e.g. seeking detailed information about a necessary medical procedure to reduce uncertainty). Thus, an important question determining the relevance of these findings for resilience to real world stressors is the extent to which controllability effects generalize across distinct types of aversive experiences and forms of control.

Animal studies of stressor controllability suggest that control over a shock stressor can blunt the behavioral effects of subsequent social stress (Amat et al., 2010). Similarly, uncontrollable social stressors have been shown to elicit the typically reported neural and behavioral consequences of uncontrollable shock stress (Gardner et al., 2005; Amat et al., 2010). These findings suggest that the effects of stressor controllability can generalize across domains. Intriguingly, evidence suggests that controllability of reward may foster vulnerability or resilience in a manner similar to control over stressors, with "free" noncontingent reward impairing subsequent instrumental reward (Overmier et al 1980) and avoidance learning (Goodkin, 1976), and operant control over reinforcement improving subsequent avoidance learning (Goodkin, 1976), increasing exploratory behavior in the open field (Joffe et al., 1973), and reducing neophobia (Mineka et al., 1986). Thus, effects of prior experience of control may have broad cross-valence behavioral generalization. Future research will be necessary to delineate the extent of this generalization and its translation to human behavior.

Our data suggest that clinical interventions involving the cultivation of a perception of control might ameliorate the excessive fear that is characteristic of anxiety disorders. The use such an instrumental "therapy" in rodents, involving completion of a series of varied controllable tasks, was effective in mitigating an uncontrollable-stress-induced impairment in subsequent avoidance learning (Williams and Maier, 1977). While some therapeutic approaches presently incorporate training in such active-coping behaviors (Van Der Kolk, 2006), the use of active-coping techniques to diminish fear in clinical practice has received significantly less attention to date than extinction-based methods. The lasting modulatory effect observed here of even a single experimental manipulation of control suggests that such techniques may represent a promising avenue for treatment (LeDoux and Gorman, 2001).

One important weakness of the present study was that a large proportion of participants assigned to the escapable stress condition failed to learn the avoidance response. This represents an important confound, as it is possible that in selecting for individuals that met this criterion, we might have also selected for individuals with a predisposition toward better fear extinction or retention. If these behaviors are indeed correlated, our apparent effect of controllable stress could actually be the result of biased participant selection. In our followup control experiment aimed at addressing this confound, we reversed the order of the learning tasks, first performing fear conditioning, and then assessing learning on the avoidance task. We found no significant difference in fear learning, extinction, and retrieval between avoidance learners and non-learners, suggesting that avoidance learning and 
extinction learning are not tightly correlated in these tasks. Moreover, comparison of learners from the original and the follow-up experiments reveal significantly improved fear extinction and retention when fear conditioning followed the avoidance session. This suggests that task order plays a critical role, supporting our interpretation that stressor controllability fostered better extinction learning and retention. Nonetheless, the inherent confound represented by the violation of random participant assignment in the initial experiment makes it particularly important that these findings be replicated in future studies that alter the stressor task in order to circumvent these learning difficulties.

In summary, research in animal models has begun to clarify the mechanisms by which stressor controllability influences subsequent fear expression (Amorapanth, LeDoux, and Nader, 2000; Maier and Watkins, 2010). Here, we take the first step towards translating this research across species by demonstrating the bidirectional effects of controllability upon conditioned fear expression in humans. Traumatic experiences are known to contribute to the development of fear-related disorders. However, some individuals appear uniquely susceptible to the influence of stress whereas others show marked resilience. These data illustrate that one aspect of our prior aversive experiences, the degree to which they are controllable, can powerfully influence the expression of learned fear. These data suggest a mechanism by which variation in life experiences might foster individual differences in the ability to persistently regulate fear expression, representing an important environmental factor underlying resilience to anxiety disorders.

\section{Acknowledgments}

This work was supported by funding from the National Institute of Mental Health grants to E.A.P (grant numbers MH062104 and MH080756) and by a National Science Foundation Graduate Research Fellowship to C.A.H. We thank Catherine Stevenson for assistance with participant testing.

\section{References}

Amat J, Aleksejev R, Paul E, Watkins L, Maier S. Behavioral control over shock blocks behavioral and neurochemical effects of later social defeat. Neuroscience. 2010; 165:1031-1038. [PubMed: 19909791]

Amat J, Baratta M, Paul E, Bland S, Watkins L, Maier S. Medial prefrontal cortex determines how stressor controllability affects behavior and dorsal raphe nucleus. Nature Neuroscience. 2005; $8: 365-371$.

Amat J, Matus-Amat P, Watkins LR, Maier SF. Escapable and inescapable stress differentially alter extracellular levels of 5-HT in the basolateral amygdala of the rat. Brain Research. 1998; 812:113120. [PubMed: 9813270]

Amat J, Paul E, Watkins L, Maier S. Activation of the ventral medial prefrontal cortex during an uncontrollable stressor reproduces both the immediate and long-term protective effects of behavioral control. Neuroscience. 2008; 154:1178-1186. [PubMed: 18515010]

Amat J, Paul E, Zarza C, Watkins L, Maier S. Previous experience with behavioral control over stress blocks the behavioral and dorsal raphe nucleus activating effects of later uncontrollable stress: role of the ventral medial prefrontal cortex. Journal of Neuroscience. 2006; 26:13264-13272. [PubMed: 17182776]

Amorapanth P, LeDoux J, Nader K. Different lateral amygdala outputs mediate reactions and actions elicited by a fear-arousing stimulus. Nature Neuroscience. 2000; 3:74-79.

Baratta M, Christianson J, Gomez D, Zarza C, Amat J, Masini C, Watkins L, Maier S. Controllable versus uncontrollable stressors bi-directionally modulate conditioned but not innate fear. Neuroscience. 2007; 146:1495-1503. [PubMed: 17478046] 
Baratta MV, Lucero TR, Amat J, Watkins LR, Maier SF. Role of the ventral medial prefrontal cortex in mediating behavioral control-induced reduction of later conditioned fear. Learning and Memory. 2008; 15:84-87. [PubMed: 18235108]

Baratta MV, Zarza CM, Gomez DM, Campeau S, Watkins LR, Maier SF. Selective activation of dorsal raphe nucleus-projecting neurons in the ventral medial prefrontal cortex by controllable stress. European Journal of Neuroscience. 2009; 30:1111-1116. [PubMed: 19686468]

Brady JV. Ulcers in“ executive” monkeys. Scientific American. 1958

Cain C, LeDoux J. Escape from fear: a detailed behavioral analysis of two atypical responses reinforced by CS termination. Journal of Experimental Psychology: Animal Behavior Processes. 2007; 33:451-463. [PubMed: 17924792]

Dawson, ME.; Schell, AM.; Filion, DL. The Electrodermal System. In: Cacioppo, JT.; Tassinary, LG.; Berntson, G., editors. Handbook of psychophysiology. 2007. p. 159-181.

Duttweiler PC. The Internal Control Index: A Newly Developed Measure of Locus of Control. Educational and Psychological Measurement. 1984; 44:209-221.

Gardner KL, Thrivikraman KV, Lightman SL, Plotsky PM, Lowry CA. Early life experience alters behavior during social defeat: focus on serotonergic systems. Neuroscience. 2005; 136:181-191. [PubMed: 16182451]

Goodkin F. Rats learn the relationship between responding and environmental events: An expansion of the learned helplessness hypothesis. Learning and Motivation. 1976; 7:382-393.

Huys QJ, Dayan P. A Bayesian formulation of behavioral control. Cognition. 2009; 113:314-328. [PubMed: 19285311]

Joffe J, Rawson R, Mulick J. Control of their environmentreduces emotionality in rats. Science. 1973; 180:1383-84. [PubMed: 4736265]

LeDoux JE, Gorman JM. A call to action: overcoming anxiety through active coping. American Journal of Psychiatry. 2001; 158:1953-1955. [PubMed: 11729007]

Lefcourt, HM. Locus of control: Current trends in theory and research. Routledge; 1982.

Lissek S, Powers AS, McClure EB, Phelps EA, Woldehawariat G, Grillon C, Pine DS. Classical fear conditioning in the anxiety disorders: a metaanalysis, Behaviour Research and Therapy. 2005; 43:1391-1424.

Maier SF, Grahn RE, Kalman BA, Sutton LC, Wiertelak EP, Watkins LR. The role of the amygdala and dorsal raphe nucleus in mediating the behavioral consequences of inescapable shock. Behavioral Neuroscience. 1993; 107:377. [PubMed: 8484901]

Maier SF, Grahn RE, Watkins LR. 8-OH-DPAT microinjected in the region of the dorsal raphe nucleus blocks and reverses the enhancement of fear conditioning and interference with escape produced by exposure to inescapable shock. Behavioral Neuroscience. 1995; 109:404. [PubMed: 7662151]

Maier SF, Watkins LR. Stressor controllability and learned helplessness: the roles of the dorsal raphe nucleus, serotonin, and corticotropin-releasing factor. Neuroscience \& Biobehavioral Reviews. 2005; 29:829-841. [PubMed: 15893820]

Maier SF, Watkins LR. Role of the medial prefrontal cortex in coping and resilience. Brain Research. 2010; 1355:52-60. [PubMed: 20727864]

Milad MR, Quirk GJ. Fear extinction as a model for translational neuroscience: ten years of progress. Annual Review of Psychology. 2012; 63:129-151.

Mineka S, Gunnar M, Champoux M. The effects of control in the early social and emotional development of rhesus monkeys. Child Development. 1986; 57:1241-1256.

Mineka S, Hendersen RW. Controllability and predictability in acquired motivation. Annual Review of Psychology. 1985; 36:495-529.

Mineka S, Zinbarg R. A contemporary learning theory perspective on the etiology of anxiety disorders: It's not what you thought it was. American Psychologist. 2006; 61:10-26. [PubMed: 16435973]

Moscarello JM, LeDoux JE. Active avoidance learning requires prefrontal suppression of amygdalamediated defensive reactions. The Journal of Neuroscience. 2013; 33:3815-3823. [PubMed: 23447593] 
Overmier, JB.; Patterson, J.; Wielkiewicz, RM. Environmental contingencies as sources of stress in animals. In: Levine, S.; Ursin, H., editors. Coping and health. Plenum Press; Cambridge, MA: MIT Press; 1980.

Peyron C, Petit JM, Rampon C, Jouvet M, Luppi PH. Forebrain afferents to the rat dorsal raphe nucleus demonstrated by retrograde and anterograde tracing methods. Neuroscience. 1998; 82:443-468. [PubMed: 9466453]

Quirk GJ, Mueller D. Neural Mechanisms of Extinction Learning and Retrieval, Neuropsychopharmacology. 2008; 33:56-72.

Rau V, DeCola JP, Fanselow MS. Stress-induced enhancement of fear learning: an animal model of posttraumatic stress disorder. Neuroscience \& Biobehavioral Reviews. 2005; 29:1207-1223. [PubMed: 16095698]

Rotter JB. Generalized expectancies for internal versus external control of reinforcement. Psychological monographs: General and applied. 1966; 80:1. [PubMed: 5340840]

Seligman M, Maier S. Failure to escape traumatic shock. Journal of Experimental Psychology. 1967; 74:1-9. [PubMed: 6032570]

Sierra-Mercado D, Padilla-Coreano N, Quirk GJ. Dissociable roles of prelimbic and infralimbic cortices, ventral hippocampus, and basolateral amygdala in the expression and extinction of conditioned fear. Neuropsychopharmacology. 2011; 36:529-538. [PubMed: 20962768]

Van Der Kolk BA. Clinical Implications of Neuroscience Research in PTSD. Annals of the New York Academy of Sciences. 2006; 1071:277-293. [PubMed: 16891578]

Weiss J, Simson PG. Depression in an animal model: Focus on the locus coeruleus. Antidepressants and receptor function. 1986; 123:191-215.

Williams JL, Maier SF. Transituational immunization and therapy of learned helplessness in the rat. Journal of Experimental Psychology: Animal Behavior Processes. 1977; 3:240-252. 


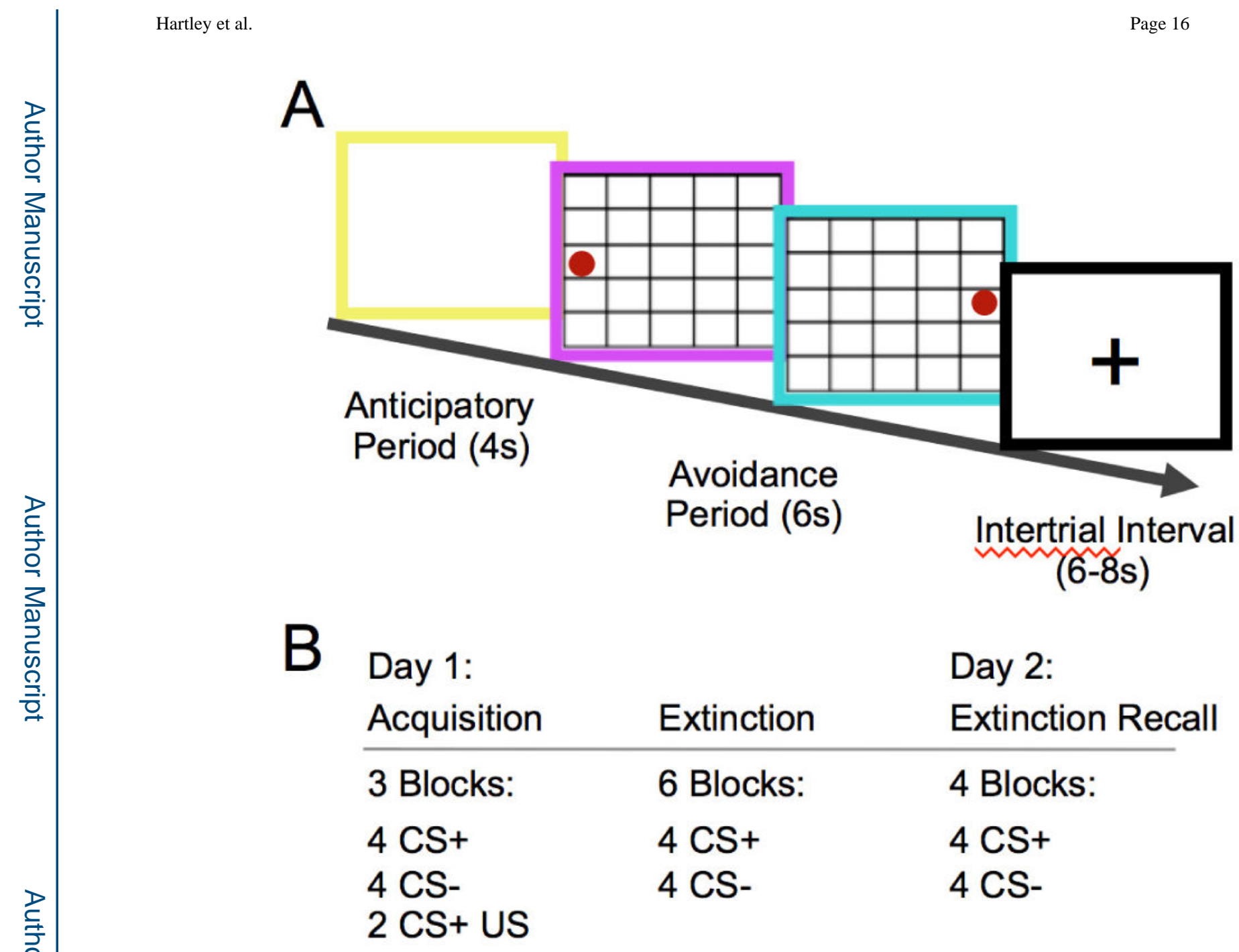

Figure 1.

Experimental paradigm A. Sequence of events during a single trial of the stressor task. Each trial began with an anticipatory period (yellow frame), followed by an avoidance period (magenta frame) during which subjects received shocks every $2 \mathrm{~s}$ until the ball was moved to the edge of the grid opposite its starting position (cyan frame). Each trial was followed by an intertrial interval. B. Phases of the two-day fear conditioning paradigm that occurred one week following the stressor task. 


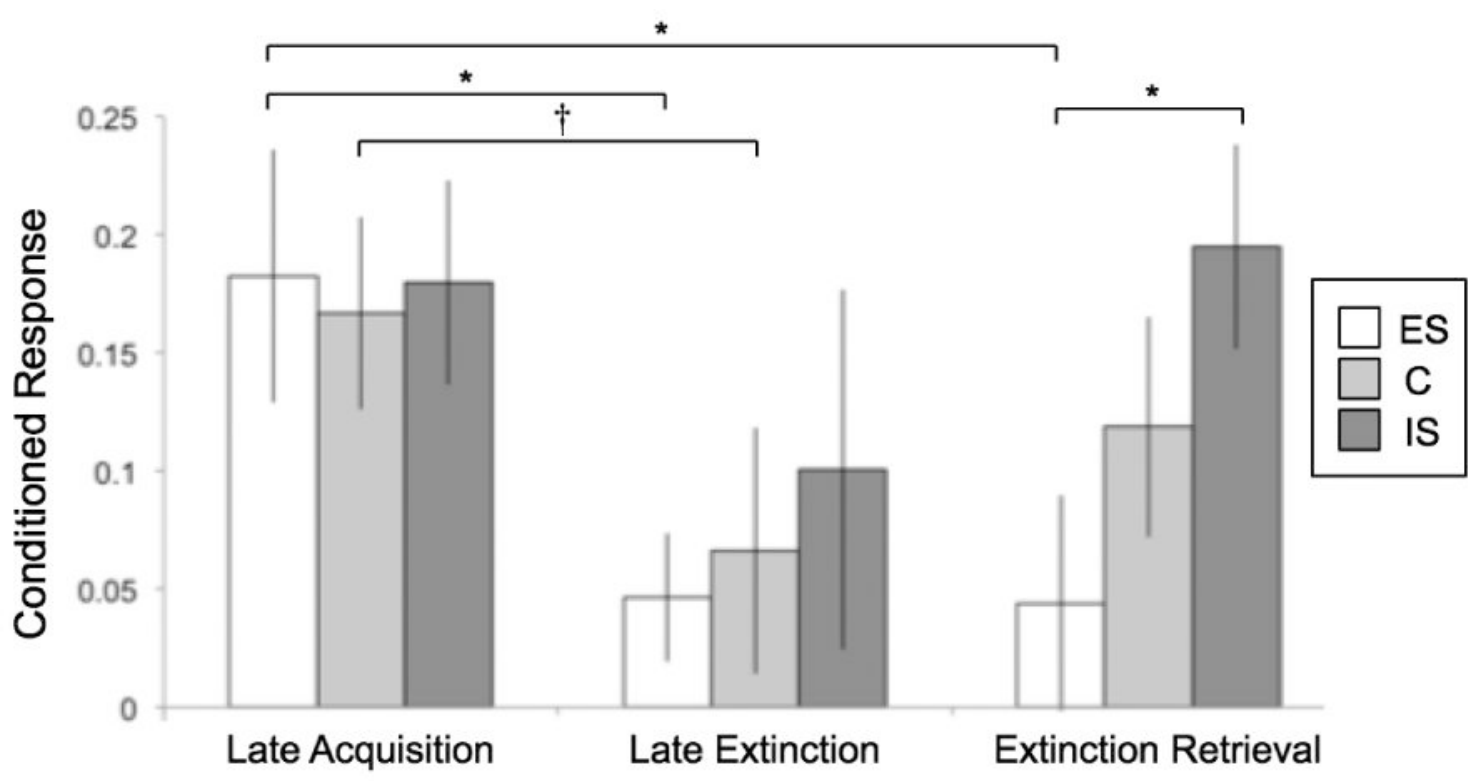

Figure 2.

Mean conditioned responses (CR; skin conductance response to CS+ minus CS-) during late acquisition, late extinction, and a second day extinction retrieval test for participants previously exposed to escapable (ES) or inescapable (IS) stress, and control (C) participants. ES and C participants, but not IS, exhibited decreased CRs during extinction. At day two retrieval test, ES fear expression remained low, C participants showed evidence of spontaneous recovery, and IS CRs increased and were significantly higher than those of ES participants. $* \mathrm{p}<.05, \dagger \mathrm{p}<.1$. 


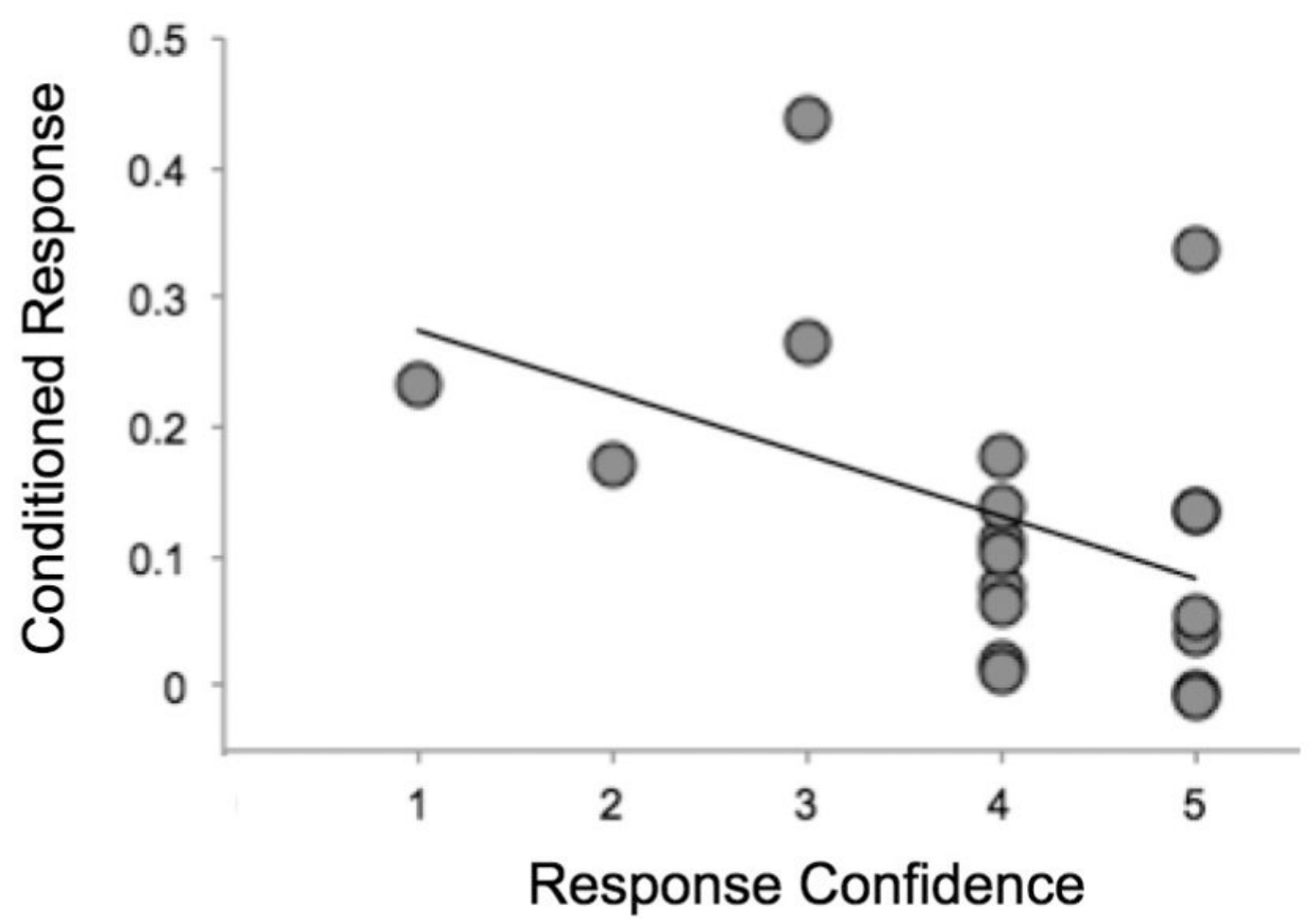

Figure 3.

ES participants reporting greater confidence that they had learned the correct avoidance response had a lower mean conditioned response across all blocks of the fear conditioning paradigm. 


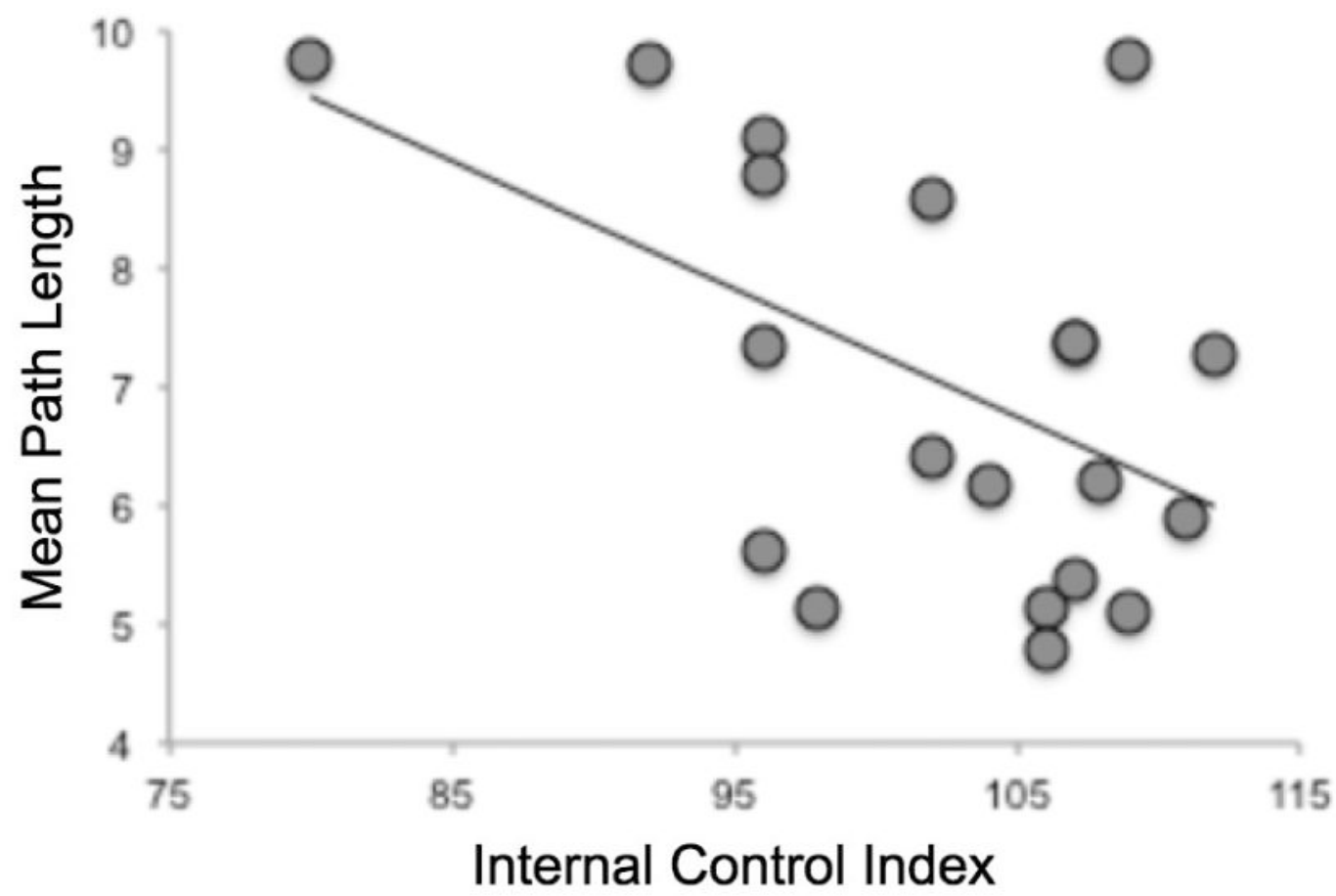

Figure 4.

ES participants with higher scores on the Internal Control Index, denoting a greater belief that reinforcement in everyday life is controllable, exhibited lower mean path lengths in avoidance learning trials, where an optimal response path of four steps could be taken to avoid shock delivery. 